\section{Neue Heilmittelrichtlinie senkt Regressgefahr ab 1. Januar 2017}

_ Der Gemeinsame Bundesausschuss (G-BA) hat das Verfahren für den langfristigen Heilmittelbedarf überarbeitet. Die Diagnoseliste wurde ergänzt (Tab. 1) und steht nun in der Anlage 2 der Heilmittelrichtlinie. Verordnungen für die darin aufgeführten Erkrankungen unterliegen nicht der Wirtschaftlichkeitsprüfung. Der Beschluss soll nach Prüfung durch das Gesundheitsministerium zum 1. Januar 2017 in Kraft treten - zeitgleich mit der Neuordnung der Wirtschaftlichkeitsprüfung ärztlich verordneter Leistungen.

\section{MMW-KOMMENTAR}

Mit den Änderungen der Heilmittelrichtlinie soll vor allem eine einheitliche Genehmigungspraxis der Krankenkassen und eine medizinische Auseinandersetzung mit den patientenindividuellen Anträgen befördert werden. Dafür gibt es eine Reihe neuer Ver- fahrensregeln. So gibt es ab 2017 kein gesondertes Genehmigungsverfahren mehr für langfristigen Heilmittelbedarf bei Erkrankungen gemäß der vorgegebenen Diagnoseliste. Bei diesen Krankheiten geht man automatisch davon aus, dass ein langfristiger Heilmittelbedarf im Sinne von $\S 32$ Abs. 1a SGB V vorliegt. Die Kasse kann hier dann nicht mehr auf einem gesonderten Genehmigungsverfahren bestehen.

Für Diagnosen, die nicht auf der Liste stehen, können Patienten weiterhin individuelle Anträge bei ihrer Kasse stellen. Die Bedingungen für die Genehmigung eines solchen Antrags sind jetzt aber genauer definiert. Die Schwere und Langfristigkeit kann sich dabei auch aus der Summe mehrerer einzelner funktioneller bzw. struktureller Schädigungen und Beeinträchtigungen der individuellen Aktivitäten ergeben, die für sich allein die Kriterien nicht erfüllen, insgesamt betrachtet jedoch einen Therapiebedarf begründen, der

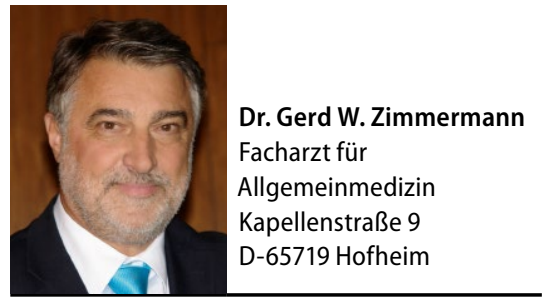

hinsichtlich Dauer und Umfang auch bei den Diagnosen der Positivliste zu erwarten wäre. Als langfristig gilt ein Therapiebedarf, wenn der Patient mindesten ein Jahr auf Heilmittel angewiesen ist.

Sofern es bei der Genehmigungsentscheidung zusätzlichen medizinischen Sachverstands bedarf, hat die Krankenkasse den Medizinischen Dienst der Krankenversicherung (MDK) einzubeziehen. Dabei müssen Therapiebedarf, -fähigkeit, -ziele und -prognose des Versicherten berücksichtigt werden. Eine Genehmigung darf nicht allein deswegen versagt werden, weil sich das Heilmittel oder die Behandlungsfrequenz im Genehmigungszeitraum ändern kann.

Die neue Anlage 2 der Heilmittelrichtlinie kann man sich im Internet ansehen. Unter www.g-ba.de/informationen/beschluesse wählt man den Unterbereich "Veranlasste Leistungen" an. Das Beschlussdatum ist der 19. Mai 2016.

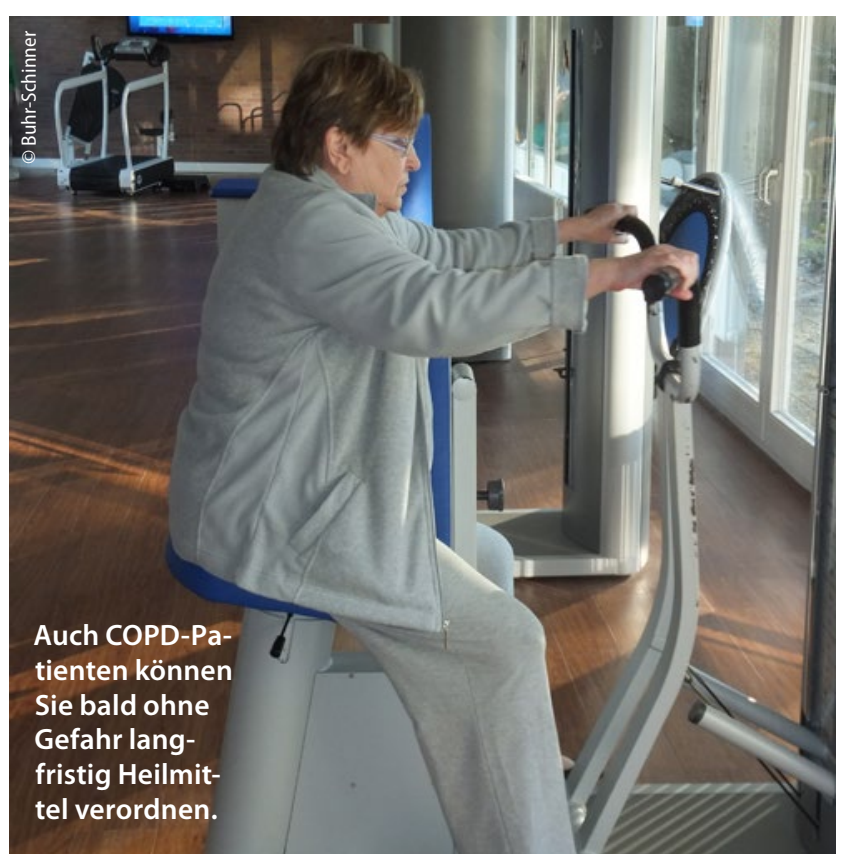

\section{Langfristiger Heilmittelbedarf: neue Diagnosen}

Diese Einträge ergänzen ab dem 1. Januar 2017 die Liste der Diagnosen, die einen langfristigen Heilmittelbedarf rechtfertigen.

- Torticollis spasticus (ICD-10-Code: G24.3)

- Syringomyelie und Syringobulbie (G95.0)

- Systemischer Lupus erythematodes (M32.1/M32.8)

- Thalidomid-Embryopathie (Q86.80)

- Angeborene Fehlbildungssyndrome mit vorwiegender Beteiligung des Gesichts (Q87.0)

- Deletion des kurzen Arms des Chromosoms 5 (Q93.4)

- Fragiles-X Chromosom (Q99.2)

- Chronische obstruktive Lungenkrankheiten (J44.00/J44.10/J44.80/J44.90)

Wichtig: Ab 1. Januar 2017 müssen Vertragsärzte für die Heilmittelverordnung elektronische Programme nutzen, die Informationen über die Heilmittelrichtlinie und über besondere Verordnungsbedarfe enthalten. Die Praxisverwaltungssysteme müssen ab diesem Zeitpunkt angepasst werden. Sie liefern dann gewissermaßen automatisch die notwendigen Informationen aus der Heilmittelrichtlinie, darunter auch die Diagnosen bei langfristigem Heilmittelbedarf. 